\title{
Possible symmetries of the superconducting order parameter in a hexagonal ferromagnet
}

\author{
K. V. Samokhin \\ Department of Physics, Brock University, St.Catharines, Ontario, Canada L2S 3A1
}

(Dated: November 9, 2018)

\begin{abstract}
We study the order parameter symmetry in a hexagonal crystal with co-existing superconductivity and ferromagnetism. An experimental example is provided by carbon-based materials, such as graphite-sulfur composites, in which an evidence of such co-existence has been recently discovered. The presence of a non-zero magnetization in the normal phase brings about considerable changes in the symmetry classification of superconducting states, compared to the non-magnetic case.

PACS numbers: 74.20.Rp, 74.70.Wz, 74.25.Ha
\end{abstract}

The recent discoveries of superconductivity co-existing with ferromagnetism in such transition element compounds as $\mathrm{ZrZn}_{2}$ [1] and $\mathrm{UGe}_{2}$ [2] have called for a revision of our views on the interplay of the two phenomena. Symmetry considerations can help us to identify the possible order parameters, even if the microscopic mechanism of pairing is not firmly established, which is often the case for the systems with strong electron correlations. The standard group-theoretical classification of the superconducting states in crystals [3] (for a review, see Ref. (4) is not applicable if the normal state is ferromagnetic. In Refs. [5], the symmetry analysis was extended to cover the magnetic case, and also a complete classification of the superconducting states in a cubic ferromagnet such as $\mathrm{ZrZn}_{2}$ was done. The orthorhombic symmetry, which is appropriate for $\mathrm{UGe}_{2}$, was studied in Refs. [6].

Very recently, a strong evidence was found of superconductivity apparently co-existing with ferromagnetism in yet another class of materials, namely graphite-sulfur composites [7] (see also Ref. [8]). The superconducting and ferromagnetic behavior in highly-oriented pyrolitic graphite was reported in Ref. [9]. The experimental data suggest that the superconductivity is associated with the graphite planes, whose symmetry is hexagonal. In this article, we study the possible pairing symmetries and the related nodal structures of the superconducting gap in a hexagonal ferromagnet, thus complementing the analysis in Refs. [5, 6. The details of the electronic spectrum of graphite [10], the nature of ferromagnetic and superconducting correlations, and also the fact that its single layer has semimetallic properties with some peculiar physics related to that [11], do not influence our results. We only assume that a standard BCS-Gor'kov phenomenology is applicable, i.e. the origin of superconductivity is the Cooper pairing of fermionic quasiparticles with opposite momenta, so that the order parameter is an anomalous average of the pair creation operator.

The starting point of the group-theoretical analysis is the symmetry group $\mathcal{G}$ of the normal state which is defined as a group of transformations which leave the system Hamiltonian invariant. In non-magnetic superconductors, time reversal symmetry is not broken, and $\mathcal{G}=S \times K \times U(1)$, where $S$ is the space group of the crystal, $K$ is time reversal operation, and $U(1)$ is the gauge group. In contrast, in magnetic crystals time reversal symmetry is broken, and $\mathcal{G}=S_{M} \times U(1)$, where $S_{M}$ is the magnetic space group which is a group of symmetry operations leaving both the crystal lattice (the microscopic charge density) and the magnetization density $M$ invariant [12]. For example, if there is a crystal point group rotation $R$ which transforms $\boldsymbol{M}$ to $-\boldsymbol{M}$, then the combined operation $K R$ will be an element of $S_{M}$, because the time reversal restores the original $\boldsymbol{M}$ not affecting the lattice symmetry. We assume that the spin-orbit coupling is sufficiently strong, so that the space group elements act on the orbital and spin coordinates simultaneously. As shown in Refs. 113, the symmetry analysis should be modified in the magnetic case, due to the fact that the operation $K R$ is anti-unitary.

The crystal structure of graphite is described by layers of honeycomb lattices of carbon atoms, the precise arrangement of the layers along the $z$ axis being not important for our analysis. We make the usual assumption that the subgroup of translations (which are replaced by magnetic translations in our case) does not affect the momentum dependence of the order parameter. Therefore, the space group in the paramagnetic phase can be replaced by the hexagonal point group $\mathbf{D}_{6 h}=\mathbf{D}_{6} \times \mathbf{C}_{i}$, where $\mathbf{C}_{i}=\{E, I\}$ (we assume that the space group contains the inversion operation $I$ ). The group $\mathbf{D}_{6}$ is generated by the rotations $C_{6 z}$ about the $z$ axis by an angle $\pi / 3$, and the rotations $C_{2 x}$ about the $x$ axis by an angle $\pi$. In the ferromagnetic phase, there are three possibilities for the easy direction of magnetization: $\boldsymbol{M}$ can be along the $z$ axis, the $x$ axis, or the $a$ axis, where $\hat{\boldsymbol{a}}=(\sqrt{3} / 2,1 / 2,0)$. Here we study only the first possibility; the other cases can be treated in a similar fashion. If $\boldsymbol{M}$ is along the $z$ axis, then the symmetry of the crystal is described by the magnetic group $\mathbf{D}_{6}\left(\mathbf{C}_{6}\right) \times \mathbf{C}_{i}$, which is generated by: (i) the rotations $C_{6 z}$, (ii) the operations $K C_{2 x}$ which combine the rotations $C_{2 x}$ with the time reversal, and (iii) the inversion $I$. The subgroup in parentheses (the unitary subgroup) incorporates all symmetry elements which are not multiplied by the anti-unitary operation $K C_{2 x}$, i.e. $\mathbf{D}_{6}\left(\mathbf{C}_{6}\right)=\mathbf{C}_{6}+K C_{2 x} \times \mathbf{C}_{6}$. [If $\boldsymbol{M}$ is along the $x$ or $a$ axes, then the symmetry is described by the magnetic 
group $\mathbf{D}_{2}\left(\mathbf{C}_{2}\right) \times \mathbf{C}_{i}$, where $\mathbf{D}_{2}\left(\mathbf{C}_{2}\right)=\mathbf{C}_{2}+K C_{2 z} \times \mathbf{C}_{2}$.]

In the presence of spin-orbit coupling, spin is not a good quantum number and is replaced by pseudospin. The free electron Hamiltonian $H_{0}$ in the normal state is $H_{0}=\sum_{\boldsymbol{k}}\left[\epsilon_{+}(\boldsymbol{k}) c_{\boldsymbol{k}+}^{\dagger} c_{\boldsymbol{k}+}+\epsilon_{-}(\boldsymbol{k}) c_{\boldsymbol{k}-}^{\dagger} c_{\boldsymbol{k}-}\right]$, where $\epsilon_{ \pm}(\boldsymbol{k})$ are the energy spectra for the pseudospin-up and pseudospindown sheets of the Fermi surface. The transformation properties of the single-electron states $|\boldsymbol{k},+\rangle$ and $|\boldsymbol{k},-\rangle$ with respect to the magnetic symmetry elements are the same as those of the spin eigenstates $|\boldsymbol{k}, \uparrow\rangle$ and $|\boldsymbol{k}, \downarrow\rangle$, which means that $C_{6 z} c_{\boldsymbol{k}, \pm}^{\dagger} C_{6 z}^{-1}=e^{\mp i \pi / 6} c_{C_{6 z} \boldsymbol{k}, \pm}^{\dagger}$, $\left(K C_{2 x}\right)\left(\lambda c_{\boldsymbol{k}, \pm}^{\dagger}\right)\left(K C_{2 x}\right)^{-1}= \pm i \lambda^{*} c_{-C_{2 x} \boldsymbol{k}, \pm}^{\dagger}$, and $I c_{\boldsymbol{k}, \pm}^{\dagger} I^{-1}=c_{-\boldsymbol{k}, \pm}^{\dagger}$. Here $\lambda$ is an arbitrary $c$-number (note that the combined operation $K C_{2 x}$ is anti-linear). The transformation properties of the superconducting order parameter can be derived using the mean-field approximation for the pairing Hamiltonian:

$$
H_{M F}=\frac{1}{2} \sum_{\boldsymbol{k}} \sum_{\alpha, \beta= \pm}\left[\Delta_{\alpha \beta}(\boldsymbol{k}) c_{\boldsymbol{k} \alpha}^{\dagger} c_{-\boldsymbol{k}, \beta}^{\dagger}+\text { h.c. }\right] .
$$

From the anticommutation relations for $c_{\boldsymbol{k} \alpha}$, we see that $\Delta_{++}(\boldsymbol{k})$ and $\Delta_{--}(\boldsymbol{k})$ are odd functions of $\boldsymbol{k}$, but $\Delta_{+-}(\boldsymbol{k})=-\Delta_{-+}(-\boldsymbol{k})$ does not have a definite parity.

The order parameter matrix can be cast in a more familiar form $\Delta(\boldsymbol{k})=\left(i \boldsymbol{\sigma} \sigma_{2}\right) \boldsymbol{d}(\boldsymbol{k})+\left(i \sigma_{2}\right) d_{0}(\boldsymbol{k})$, where $\boldsymbol{d}(\boldsymbol{k})=-\boldsymbol{d}(-\boldsymbol{k})$ and $d_{0}(\boldsymbol{k})=d_{0}(-\boldsymbol{k})$ are the pseudospintriplet and the pseudospin-singlet components respectively [ [ 1 . It is convenient to introduce an orthogonal basis of unit vectors $\hat{\boldsymbol{e}}_{1}, \hat{\boldsymbol{e}}_{2}, \hat{\boldsymbol{e}}_{3}$ in the pseudospin space, such that $\hat{\boldsymbol{e}}_{3} \| M$ and use the following representation of the vector order parameter: $\boldsymbol{d}(\boldsymbol{k})=\hat{\boldsymbol{e}}_{+} d_{-}(\boldsymbol{k})+\hat{\boldsymbol{e}}_{-} d_{+}(\boldsymbol{k})+$ $\hat{\boldsymbol{e}}_{3} d_{3}(\boldsymbol{k})$, where $\hat{\boldsymbol{e}}_{ \pm}=\left(\hat{\boldsymbol{e}}_{1} \pm i \hat{\boldsymbol{e}}_{2}\right) / \sqrt{2}$ and $d_{+}=\left(d_{1}+\right.$ $\left.i d_{2}\right) / \sqrt{2}=\Delta_{--} / \sqrt{2}, d_{-}=\left(d_{1}-i d_{2}\right) / \sqrt{2}=-\Delta_{++} / \sqrt{2}$, $d_{3}=\left(\Delta_{+-}+\Delta_{-+}\right) / 2$. Also, $d_{0}=\left(\Delta_{+-}-\Delta_{-+}\right) / 2$. From Eq. (1), we obtain the transformation rules for the order parameters under the magnetic group operations:

$$
\begin{aligned}
& C_{6 z} d_{ \pm}(\boldsymbol{k})=e^{ \pm i \pi / 3} d_{ \pm}\left(C_{6 z}^{-1} \boldsymbol{k}\right) \\
& C_{6 z} d_{3}(\boldsymbol{k})=d_{3}\left(C_{6 z}^{-1} \boldsymbol{k}\right) \\
& K C_{2 x} d_{ \pm}(\boldsymbol{k})=d_{ \pm}^{*}\left(C_{2 x}^{-1} \boldsymbol{k}\right) \\
& K C_{2 x} d_{3}(\boldsymbol{k})=-d_{3}^{*}\left(C_{2 x}^{-1} \boldsymbol{k}\right)
\end{aligned}
$$

and

$$
\begin{aligned}
& C_{6 z} d_{0}(\boldsymbol{k})=d_{0}\left(C_{6 z}^{-1} \boldsymbol{k}\right) \\
& K C_{2 x} d_{0}(\boldsymbol{k})=d_{0}^{*}\left(C_{2 x}^{-1} \boldsymbol{k}\right) .
\end{aligned}
$$

Because of the inversion symmetry, the triplet and the singlet order parameters can be considered separately.

Triplet order parameter. The superconducting order parameter transforms according to one of the irreducible representations $\Gamma$ of the normal state symmetry group $\mathcal{G}$. In our case, $\mathcal{G}$ contains the anti-unitary operation $K C_{2 x}$, and the standard symmetry analysis should be modified: instead of usual representations, one should use co-representations of the magnetic point group $\mathbf{D}_{6}\left(\mathbf{C}_{6}\right)$, which can be derived from one-dimensional representations of the unitary subgroup $\mathbf{C}_{6}$ [13]. The results for odd co-representations $\Gamma$ are listed in Table I. Note that the action of the unitary and anti-unitary orbital symmetry elements on scalar functions $f(\boldsymbol{k})$ is defined as follows: $R f(\boldsymbol{k})=f\left(R^{-1} \boldsymbol{k}\right)$, and $K R f(\boldsymbol{k})=f^{*}\left(-R^{-1} \boldsymbol{k}\right)$.

The basis functions of the co-representations in Table Ifvanish on some lines or planes in the momentum space. Although the specific form of the basis function is not imposed by symmetry [for example, any $f_{\Gamma}(\boldsymbol{k})$ can be multiplied by an arbitrary real function of $k_{z}^{2}, k_{x}^{2}+k_{y}^{2}$, $\left(k_{y} \pm i k_{x}\right)^{6}$, etc.], the position of the zeros in $\boldsymbol{k}$-space is independent of the choice of $f_{\Gamma}(\boldsymbol{k})$. It can be checked that the zeros of the basis functions from Table 1 are all required by symmetry, and, on the other hand, there are no other symmetry-imposed zeros of the basis functions.

All co-representations of $\mathbf{D}_{6}\left(\mathbf{C}_{6}\right)$ are one-dimensional, therefore the order parameter can be represented as

$$
\boldsymbol{d}_{\Gamma}(\boldsymbol{k})=i \eta_{-} \hat{\boldsymbol{e}}_{+} f_{\Gamma_{-}}(\boldsymbol{k})+i \eta_{+} \hat{\boldsymbol{e}}_{-} f_{\Gamma_{+}}(\boldsymbol{k})+\eta_{3} \hat{\boldsymbol{e}}_{3} f_{\Gamma_{3}}(\boldsymbol{k}) .
$$

Thus, the order parameter has three components $\eta_{+}$, $\eta_{-}$, and $\eta_{3}$, which enter the Ginzburg-Landau (GL) free energy and can be $\boldsymbol{r}$-dependent, in general. It is obvious from Eqs. (2) and (4) that the orbital symmetries of $d_{+}, d_{-}$, and $d_{3}$, which are described by the corepresentations $\Gamma_{+}, \Gamma_{-}$, and $\Gamma_{3}$ respectively, should all be different. In Table $\mathbb{I}$. we list the combinations of the orbital co-representations which give rise to the same symmetry $\Gamma$ of the vector $\boldsymbol{d}$. For example, the vector $\boldsymbol{d}(\boldsymbol{k})=i \eta_{-} \hat{\boldsymbol{e}}_{+} f_{B_{u}}(\boldsymbol{k})+i \eta_{+} \hat{\boldsymbol{e}}_{-} f_{1_{E_{1 u}}}(\boldsymbol{k})+\eta_{3} \hat{\boldsymbol{e}}_{3} f_{E_{2}}(\boldsymbol{k})$ transforms according to the co-representation ${ }^{2} E_{2 u}$.

The transformations of the pseudospin vector $\boldsymbol{d}$ under the magnetic symmetry elements can be interpreted as operations acting on $\boldsymbol{\eta}=\left(\eta_{+}, \eta_{-}, \eta_{3}\right)^{T}$. Namely, for a co-representation $\Gamma, C_{6 z} \boldsymbol{\eta}(\boldsymbol{r})=\chi_{\Gamma}\left[C_{6 z}\right] \boldsymbol{\eta}(\boldsymbol{r})$, where $\chi_{\Gamma}$ is the character of $C_{6 z}$ from Table [1. Also, because of our choice of the overall phases of the basis functions (see the caption to Table $\mathbb{1}$ ) and the presence of the factors $i$ on the right-hand side of Eqs. (雨), $K C_{2 x} \boldsymbol{\eta}(\boldsymbol{r})=\boldsymbol{\eta}^{*}(\boldsymbol{r})$. Note that there are no symmetry operations that transform, say, $\eta_{+}$to $\eta_{-}$, etc, which has profound consequences for the GL theory of ferromagnetic superconductors. Indeed, the GL free energy contains all combinations of the order parameter components and the spatial gradients that are invariant under the transformations from $\mathcal{G}$. The fact that the only change that occurs to $\boldsymbol{\eta}$ under the magnetic symmetry operations is the multiplication by a phase factor which is the same for all three components means that there are more invariant terms allowed in the GL functional for a ferromagnetic superconductor than for its non-ferromagnetic counterpart. The general form of the uniform terms in the free energy density is

$$
F=\sum_{i j= \pm, 3} A_{i j}(T) \eta_{i}^{*} \eta_{j}+\sum_{i j k l= \pm, 3} B_{i j, k l} \eta_{i}^{*} \eta_{j}^{*} \eta_{k} \eta_{l},
$$

where $A_{i j}$ is a real symmetric matrix, and the matrix $B$ is symmetric with respect to $i \leftrightarrow j$ and $k \leftrightarrow l$, and 
TABLE I: The character table and the examples of the basis functions for the odd irreducible co-representations of the magnetic point group $\mathbf{D}_{6}\left(\mathbf{C}_{6}\right)$. The overall phases of the basis functions are chosen so that $K C_{2 x} f_{\Gamma}(\boldsymbol{k})=f_{\Gamma}(\boldsymbol{k})$. $\omega=\exp (2 \pi i / 3)$, and $\lambda_{1,2}$ are arbitrary real constants.

\begin{tabular}{|c||c|c|c|}
\hline \hline$\Gamma$ & $E$ & $C_{6 z}$ & $f_{\Gamma}(\boldsymbol{k})$ \\
\hline$A_{u}$ & 1 & 1 & $k_{z}$ \\
\hline$B_{u}$ & 1 & -1 & $\lambda_{1}\left(k_{y}+i k_{x}\right)^{3}+\lambda_{2}\left(k_{y}-i k_{x}\right)^{3}$ \\
\hline${ }^{1} E_{1 u}$ & 1 & $-\omega^{*}$ & $k_{y}+i k_{x}$ \\
\hline${ }^{2} E_{1 u}$ & 1 & $-\omega$ & $k_{y}-i k_{x}$ \\
\hline${ }^{1} E_{2 u}$ & 1 & $\omega^{*}$ & $k_{z}\left(k_{y}-i k_{x}\right)^{2}$ \\
\hline${ }^{2} E_{2 u}$ & 1 & $\omega$ & $k_{z}\left(k_{y}+i k_{x}\right)^{2}$ \\
\hline \hline
\end{tabular}

TABLE II: The sets of orbital co-representations corresponding to the same symmetry of the triplet order parameter $\boldsymbol{d}_{\Gamma}(\boldsymbol{k})$ [see Eq. (⿹)].

\begin{tabular}{|c||c|}
\hline \hline$\Gamma$ & $\left(f_{\Gamma_{+}}(\boldsymbol{k}), f_{\Gamma_{-}}(\boldsymbol{k}), f_{\Gamma_{3}}(\boldsymbol{k})\right)$ \\
\hline$A_{u}$ & $\left({ }^{2} E_{1 u},{ }^{1} E_{1 u}, A_{u}\right)$ \\
\hline$B_{u}$ & $\left({ }^{2} E_{2 u},{ }^{1} E_{2 u}, B_{u}\right)$ \\
\hline${ }^{1} E_{1 u}$ & $\left(A_{u},{ }^{2} E_{2 u},{ }^{1} E_{1 u}\right)$ \\
\hline${ }^{2} E_{1 u}$ & $\left({ }^{1} E_{2 u}, A_{u},{ }^{2} E_{1 u}\right)$ \\
\hline${ }^{1} E_{2 u}$ & $\left(B_{u},{ }^{2} E_{1 u},{ }^{1} E_{2 u}\right)$ \\
\hline${ }^{2} E_{2 u}$ & $\left({ }^{1} E_{1 u}, B_{u},{ }^{2} E_{2 u}\right)$ \\
\hline \hline
\end{tabular}

satisfies the following condition: $B_{i j, k l}=B_{k l, i j}$. The critical temperature $T_{c}$ is defined as the temperature at which one of the eigenvalues of $A$ changes sign. At $T>$ $T_{c}, A$ is positive definite, and $\eta_{+}=\eta_{-}=\eta_{3}=0$. Below $T_{c}$, all three components of $\boldsymbol{\eta}$ are non-zero, in general. In addition to Eq. (5), the GL functional contains a variety of the gradient terms. All this can lead to a rich phase diagram, which we shall not discuss here. Let us just note Eq. (5) is formally equivalent to a model of a threeband superconductor with three scalar order parameters of the same symmetry.

An important consequence of the above results is that the gap in the spectrum of Bogoliubov quasiparticles goes to zero at some points or lines at the Fermi surface. The excitation spectrum can be obtained by diagonalizing the Hamiltonian $H=H_{0}+H_{M F}$. The quasiparticle energy $E(\boldsymbol{k})$ vanishes at some $\boldsymbol{k}$ if the following condition is satisfied:

$$
\begin{array}{r}
\epsilon_{+}^{2} \epsilon_{-}^{2}+2 \epsilon_{+}^{2}\left|d_{+}\right|^{2}+2 \epsilon_{-}^{2}\left|d_{-}\right|^{2}+2 \epsilon_{+} \epsilon_{-}\left|d_{3}\right|^{2} \\
+\left|2 d_{+} d_{-}+d_{3}^{2}\right|^{2}=0 .
\end{array}
$$

Thus, the condition for the gap zeros on the pseudospinup sheet of the Fermi surface [at $\epsilon_{+}(\boldsymbol{k})=0$ ], is that $d_{-}(\boldsymbol{k})=d_{3}(\boldsymbol{k})=0$, i.e. $f_{\Gamma_{-}}(\boldsymbol{k})=f_{\Gamma_{3}}(\boldsymbol{k})=0$. For the gap zeros on the pseudospin-down sheet $\left[\right.$ at $\left.\epsilon_{-}(\boldsymbol{k})=0\right]$, we must have $d_{+}(\boldsymbol{k})=d_{3}(\boldsymbol{k})=0$, i.e. $f_{\Gamma_{+}}(\boldsymbol{k})=f_{\Gamma_{3}}(\boldsymbol{k})=$ 0 . Using Tables [1] and [I], we see that if the symmetry of $\boldsymbol{d}$ corresponds to the co-representations $B_{u},{ }^{1} E_{2 u}$, or
${ }^{2} E_{2 u}$, then all three orbital basis functions have zeros on the line $k_{x}=k_{y}=0$, so that the energy gap vanishes at the poles of both sheets of the Fermi surface. On the contrary, for the ${ }^{1} E_{1 u}$ and ${ }^{2} E_{1 u}$ symmetries, the gap goes to zero at the poles of one of the sheets, while the other sheet remains fully gapped. For the $A_{u}$ symmetry, there are no symmetry-imposed gap zeros.

In the discussion above, we implicitly assumed that all three components of $\boldsymbol{d}$ have comparable magnitude. More realistic scenario is that the conditions for the appearance of superconductivity are more favorable on one of the sheets of the Fermi surface, so that only one component of the order parameter, say $d_{-}$on the pseudospin-up sheet, is dominant. Then, the $d_{+}$component is induced by the inter-band interactions of the form $c_{\boldsymbol{k}+}^{\dagger} c_{-\boldsymbol{k},+}^{\dagger} c_{\boldsymbol{k}^{\prime}-} c_{-\boldsymbol{k}^{\prime},-}$, which are expected to be small if the spin-orbit coupling is weak (these interactions vanish at zero spin-orbit coupling because of the spin conservation). Also, if the exchange band splitting greatly exceeds the superconducting energy scale, which is of the order of $T_{c}$, then the inter-band interactions $c_{\boldsymbol{k}+}^{\dagger} c_{-\boldsymbol{k},-}^{\dagger} c_{\boldsymbol{k}^{\prime}-} c_{-\boldsymbol{k}^{\prime},+}$ responsible for the $d_{3}$-component, are negligibly small. While this is the case in such materials as $\mathrm{ZrZn}_{2}$ and $\mathrm{UGe}_{2}$, it is not clear whether it is also true for the graphite-based ferromagnets. If the $d_{3}$-component can indeed be neglected, then the conditions for the presence of the gap zeros become less restrictive, so that, as seen from Tables 1 and 1 . the energy gap always has either line and/or point nodes on both sheets of the Fermi surface.

Singlet order parameter. Similar to the $d_{3}$-component of the triplet pairing, the singlet pairing can only be realized if the exchange band splitting is less than the superconducting $T_{c}$ (Chandrasekhar-Clogston limit) 14]. This limit can be slightly exceeded if to consider the possibility of the Cooper pairs having a non-zero momentum (Larkin-Ovchinnikov-Fulde-Ferrell state) 15. The symmetry analysis can be done similarly to the triplet case. The only difference is that there is only one order parameter component, and the symmetry is described by even co-representations of $\mathbf{D}_{6}\left(\mathbf{C}_{6}\right)$. We have

$$
d_{0, \Gamma}(\boldsymbol{k})=\psi f_{\Gamma}(\boldsymbol{k}),
$$

where $\psi$ is a quantity which enters the GL functional, and $f_{\Gamma}(\boldsymbol{k})$ is the basis function for the co-representation $\Gamma$, see Table III. As mentioned above, $f_{\Gamma}(\boldsymbol{k})$ can be multiplied by an arbitrary real function of $k_{z}^{2}, k_{x}^{2}+k_{y}^{2},\left(k_{y} \pm i k_{x}\right)^{6}$, etc. Under the action of the magnetic symmetry elements, $C_{6 z} \psi(\boldsymbol{r})=\chi_{\Gamma}\left[C_{6 z}\right] \psi(\boldsymbol{r})$, where $\chi_{\Gamma}$ is the character of $C_{6 z}$ from Table III, and also $K C_{2 x} \psi(\boldsymbol{r})=\psi^{*}(\boldsymbol{r})$.

The condition for the gap in the excitation energy $E(\boldsymbol{k})$ to vanish at some $\boldsymbol{k}$ is simply

$$
\epsilon_{+} \epsilon_{-}+\left|d_{0}\right|^{2}=0,
$$

therefore the gap nodes appear simultaneously on both sheets of the Fermi surface where the basis function $f_{\Gamma}(\boldsymbol{k})$ has symmetry-imposed zeros. From Table III, we see that for all order parameter symmetries, except from $A_{g}$, the 
TABLE III: The character table and the examples of the basis functions for the even irreducible co-representations of the magnetic point group $\mathbf{D}_{6}\left(\mathbf{C}_{6}\right)$. For all $\Gamma, K C_{2 x} f_{\Gamma}(\boldsymbol{k})=f_{\Gamma}(\boldsymbol{k})$, and $\lambda_{1,2}$ are arbitrary real constants.

\begin{tabular}{|c||r|c|c|}
\hline \hline$\Gamma$ & $E$ & $C_{6 z}$ & $f_{\Gamma}(\boldsymbol{k})$ \\
\hline$A_{g}$ & 1 & 1 & 1 \\
\hline$B_{g}$ & 1 & -1 & $k_{z}\left[\lambda_{1}\left(k_{y}+i k_{x}\right)^{3}+\lambda_{2}\left(k_{y}-i k_{x}\right)^{3}\right]$ \\
\hline${ }^{1} E_{1 g}$ & 1 & $-\omega^{*}$ & $k_{z}\left(k_{y}+i k_{x}\right)$ \\
\hline${ }^{2} E_{1 g}$ & 1 & $-\omega$ & $k_{z}\left(k_{y}-i k_{x}\right)$ \\
\hline${ }^{1} E_{2 g}$ & 1 & $\omega^{*}$ & $\left(k_{y}-i k_{x}\right)^{2}$ \\
\hline${ }^{2} E_{2 g}$ & 1 & $\omega$ & $\left(k_{y}+i k_{x}\right)^{2}$ \\
\hline \hline
\end{tabular}

gap goes to zero either on the equators or at the poles of both Fermi surfaces.

The expressions for the basis functions of the corepresentations of $\mathbf{D}_{6}\left(\mathbf{C}_{6}\right)$ given in Tables II and III are applicable if the Fermi surface is centered around the $\Gamma$ point of the first Brillouin zone. It is not the case in graphite, where the Fermi surface consists of small "sausage"-like pockets along the six vertical edges of the hexagonal Brillouin zone, i.e. along the lines $\boldsymbol{k}=C_{6 z}^{n} \boldsymbol{k}_{s}$, where $n=0, \ldots, 5, \boldsymbol{k}_{s}=2 \boldsymbol{K}_{1} / 3-\boldsymbol{K}_{2} / 3+k_{z} \hat{\boldsymbol{e}}_{z}$, and $\boldsymbol{K}_{1}$ and $\boldsymbol{K}_{2}$ are the reciprocal lattice vectors [10]. It can be easily proved that for all odd and even $E$ corepresentations, in addition to the zeros at $k_{x}=k_{y}=0$, the basis functions also vanish at the vertical edges of the Brillouin zone, because $\boldsymbol{k}_{s}$ and $C_{3 z} \boldsymbol{k}_{s}$ are equivalent points. Similarly, if a basis function vanishes on the plane $k_{z}=0$, then it should also vanish at the horizontal surfaces of the Brillouin zone, i.e. at $k_{z}= \pm \pi / c_{0}$, where $c_{0}$ is the lattice constant of graphite along the $z$ axis.

The presence of the gap nodes would manifest itself in power-law temperature dependences of the thermodynamic and transport properties [1]. For example, the electronic specific heat at low temperatures should be $C(T) / T \sim T^{2}$ for the first-order point nodes, $C(T) / T \sim$ $T$ for the line nodes or the second-order point nodes, and $C(T) / T \sim T^{2 / 3}$ for the third-order point nodes.

To summarize, we have derived a complete symmetry classification of the superconducting states in a hexagonal ferromagnet, considering both the triplet and the singlet pairing channels. We have discussed the nodal structure of the gap in the excitation spectrum, and also the modifications to the Ginzburg-Landau theory for ferromagnetic superconductors. So far, the only experimental system to which our results might be applicable is the graphite-sulfur composite studied in Ref. [7.

The author has greatly benefited from the numerous discussions with M. Walker about the symmetry of ferromagnetic superconductors. The author thanks B. Mitrovic for useful comments and interest to this work, and also F. Razavi and M. Reedyk for the discussions of the experimental situation. The financial support from Brock University is gratefully acknowledged.
[1] C. Pfleiderer, M. Uhlarz, S. M. Hayden, R. Vollmer, H. v. Löhneysen, N. R. Bernhoeft, and G. G. Lonzarich, Nature 412, 58 (2001).

[2] S. S. Saxena, P. Agarwal, K. Ahilan, F. M. Grosche, R. K. W. Haselwimmer, M. J. Steiner, E. Pugh, I. R. Walker, S. R. Julian, P. Monthoux, G. G. Lonzarich, A. Huxley, I. Sheikin, D. Braithwaite, and J. Flouquet, Nature 406, 587 (2000).

[3] G. E. Volovik and L. P. Gor'kov, Sov. Phys. - JETP 61, 843 (1985) [Zh. Eksp. Teor. Fiz. 88, 1412 (1985)]; K. Ueda and T. M. Rice, Phys. Rev. B 31, 7114 (1985); E. I. Blount, Phys. Rev. B 32, 2935 (1985).

[4] V. P. Mineev and K. V. Samokhin, Introduction to Unconventional Superconductivity (Gordon and Breach, The Netherlands, 1999).

[5] M. B. Walker and K. V. Samokhin, Phys. Rev. Lett. 88, 207001 (2002); K. V. Samokhin and M. B. Walker, Phys. Rev. B 66, 024512 (2002); K. V. Samokhin and M. B. Walker, arXiv:cond-mat/0206487.

[6] V. P. Mineev, arXiv:cond-mat/0204263; I. A. Fomin, arXiv:cond-mat/0207152 (see also I. A. Fomin, JETP Lett. 74, 111 (2001) [Pis'ma Zh. Eksp. Teor. Fiz. 74, 116 (2001)], for an earlier attempt of the symmetry analysis for $\mathrm{UGe}_{2}$ ).

[7] S. Moehlecke, P.-C. Ho, and M. B. Maple, arXiv:condmat/0204006.
[8] R. Ricardo da Silva, J. H. S. Torres, and Y. Kopelevich, Phys. Rev. Lett. 87, 147001 (2001).

[9] Y. Kopelevich, P. Esquinazi, J. H. S. Torres, and S. Moehlecke, J. Low Temp. Phys. 119, 691 (2000).

[10] M. S. Dresselhaus and J. G. Mavroides, IBM J. Res. Develop. 8, 262 (1964); S. J. Williamson, S. Foner, and M. S. Dresselhaus, Phys. Rev. 140, A1429 (1965).

[11] J. Gonzalez, F. Guinea, and M. A. H. Vozmediano, Phys. Rev. B 63, 134421 (2001); D. V. Khveshchenko, Phys. Rev. Lett. 87, 246802 (2001); G. Baskaran and S. A. Jafari, Phys. Rev. Lett. 89, 016402 (2002).

[12] L. D. Landau and E. M. Lifshitz, Electrodynamics of Continuous Media (Pergamon Press, London, 1960).

[13] E. Wigner, Group Theory and Its Applications to the Quantum Mechanics of Atomic Spectra (Academic Press, NY, 1959); C. J. Bradley and A. P. Cracknell, The Mathematical Theory of Symmetry in Solids (Clarendon Press, Oxford, 1972); A. P. Cracknell, Progr. Theor. Phys. 35, 196 (1966).

[14] B. S. Chandrasekhar, Appl. Phys. Lett. 1, 7 (1962); A. M. Clogston, Phys. Rev. Lett. 9, 266 (1962).

[15] A. I. Larkin and Yu. N. Ovchinnikov, Sov. Phys. - JETP 20, 762 (1965) [Zh. Eksp. Teor. Fiz. 47, 1136 (1964)]; P. Fulde and R. A. Ferrell, Phys. Rev. 135, 550 (1964). 